\title{
Inversión necesaria, urgente y asequible en salud mental. Propuestas de FEAFES
}

\author{
An unavoidable, urgent and affordable \\ investment in mental health. Proposals \\ FEAFES
}

\section{Palabras clave:}

Trastorno mental, medidas

terapéuticas, sociosanitario, derechos.

\section{Keywords}

Mental illness, therapeutic measures, sociosanitary, rights.

\section{Introducción}

El Comisario para los Derechos Humanos del Consejo de Europa, Nils Muižnieks, visitó España en junio de 20I 3 y conoció la situación de las personas con discapacidad a causa de trastorno mental (referida por él como "discapacidad psicosocial”). En su informe (Muižnieks, 20I3: 26, 27, 29, 30) instó a las autoridades españolas a "no escatimar esfuerzos para evitar que la falta de recursos se traduzca en la reinstitucionalización de estas personas", ya que "esto constituiría un enorme retroceso". Para que estas personas puedan "lograr una mayor autonomía e inclusión social”, reclamó un mayor "esfuerzo para garantizar el acceso al apoyo apropiado”. El Comisario se preocupó por el impacto que en estas personas están teniendo las políticas de "recortes en las subvenciones destinadas a servicios y cuidados" de los últimos años.

En enero de 20I4, el Instituto Nacional de Estadística (INE) publicó los datos de defunciones según la causa de muerte de 2012 destacando el suicidio como "principal causa externa de mortalidad" (INE, 20I4: 7) con una tasa de 7' 6 por cada I000.000 personas (la tasa más alta desde 2005) produciéndose un

\section{José Ma Sánchez Monge <comunicacion@feafes.org>}

Presidente de la Confederación Española de Agrupaciones de Familiares y Personas con Enfermedad Mental (FEAFES)
Para citar:

Sánchez Monge, J.M. (20I4):

"Inversión necesaria, urgente y asequible en salud mental. Propuestas de FEAFES”. Revista Española de Discapacidad, 2 (I): 2I 5-22I.

$<$ http://dx.doi.org/IO.5569/23405104.02.01.I3> 
incremento del I I’3 \% frente al año anterior. Llama también la atención que entre los grupos que experimentaron un mayor incremento de defunciones se encuentran los trastornos mentales y del comportamiento, con un I 2,2 \% más que en 2OI I (INE, 20I4: 4).

La propia Organización Mundial de la Salud (OMS), ha cuestionado que "a pesar de la necesidad simultánea de más servicios sociales y de salud mental debido al aumento de las tasas de trastornos mentales y suicidio", se hayan generado "recortes en la financiación" (OMS, 2013: 7).

Esta organización ha señalado (OMS, 20I3: 8) que las tasas de discapacidad y mortalidad entre las personas con trastorno mental son "desproporcionadamente elevadas". La probabilidad de "muerte prematura es un 40-60\% mayor entre las personas con depresión mayor o esquizofrenia que la población general, debido a los problemas de salud física" que no son atendidos correctamente. Por sí sola, la depresión representa un 4,3\% de la carga mundial de morbilidad y se encuentra entre las principales causas mundiales de discapacidad (un I I \% del total mundial de años vividos con discapacidad).

Por otra parte, el impacto del recorte en las ayudas y prestación de servicios a las personas en situación de dependencia y para la promoción de la autonomía personal, el incompleto desarrollo e implantación de la Estrategia en Salud Mental del Sistema Nacional de Salud (ESM) en todas las comunidades autónomas, la necesaria y obligatoria adaptación de nuestra normativa a la Convención Internacional sobre los Derechos de las Personas con Discapacidad (CIDPD), el aparente aumento de los problemas de salud mental ${ }^{\mathrm{r}}$ y recrudecimiento de las

I. Así lo apuntan diferentes estudios, expertos y profesionales del ámbito de la salud mental: "Una crisis que trastorna" (<http://ccaa.elpais.com/ccaa/20I3/Io/ı 8/andalucia/I382I I2436_58I64I.html>; 20 de octubre de 20I3); "Aumento considerable de trastornos mentales en las consultas de Atención Primaria” (<http://www.infocop.es/view_article. asp?id=4430>; I de marzo de 20I3); "Estamos muertos y tenemos miedo a resucitar" (<http://ccaa.elpais.com/ccaa/20I3/05/o8/ valencia/I368038203_835095.html>; 9 de mayo de 20I3). condiciones de vida de las personas con trastorno mental y el peligro de que se recurra a modelos anteriores al comunitario por resultar aparentemente más económicos y de más sencilla aplicación, llevan a pensar que es preciso reforzar las políticas públicas de promoción de la salud mental, prevención y atención a los trastornos mentales de base comunitaria.

\section{Salud mental: hacia el bienestar global}

Para la OMS la salud es "un estado de completo bienestar físico, mental y social, y no solamente la ausencia de afecciones o enfermedades". Considera que la salud mental es "un estado de bienestar en el cual el individuo es consciente de sus propias capacidades, puede afrontar las tensiones normales de la vida, puede trabajar de forma productiva y fructífera y es capaz de hacer una contribución a su comunidad".

Nuestra Carta Magna, a la que a tantas personas gusta nombrar, estableció como principios rectores de la política social y económica que los poderes públicos asegurasen la protección a la salud, la asistencia y prestaciones sociales, y promovieran las condiciones favorables para el progreso social.

Es dentro de este marco, internacional y nacional, donde debemos situar las políticas de promoción de la salud mental y de atención al trastorno mental, entendidas como estrategias que garanticen el desarrollo de una vida digna y plena para cualquier persona. Sin embargo, nos encontramos con recortes en prestaciones sociales, el fin de la asistencia sanitaria universal y gratuita, políticas educativas austeras, medidas fallidas frente al desempleo, precariedad laboral y obstáculos, a veces insalvables, para acceder a una vivienda. Todo ello al albor de la crisis económica que se ha convertido en un argumento, no por más veces nombrado más correcto, para justificar lo que, entendemos, carece de cualquier respaldo en materia de derechos económicos, sociales y culturales, independientemente de si de los mismos son 
sujetos las personas con buena, o mala, salud mental.

En España, después de años de ostracismo y con un atraso considerable con respecto a los países de nuestro entorno, la atención a las personas con enfermedad mental cambió radicalmente su curso tras la entrada en vigor de la Ley General de Sanidad de $1986^{2}$.

Casi 30 años después aún se sigue hablando de cómo articular lo establecido en esta ley, seguimos denunciando la falta de recursos y seguimos esforzándonos por acabar con el estigma, el desconocimiento y la incomprensión hacia las personas con trastorno mental, quizás las principales motivaciones que pueden estar detrás de recientes turbulencias sufridas.

Para alcanzar el objetivo planteado por la OMS sobre "proporcionar en el ámbito comunitario servicios de asistencia social y de salud mental completos, integrados y con capacidad de respuesta" (OMS, 20I3: I4) y con el concepto de participación por bandera, consideramos que la base de la que deben emanar todas las actuaciones en esta materia es la respuesta a las necesidades de las personas con trastorno mental y sus familias. Cabe destacar: recibir información respeto a sus derechos fundamentales y a su capacidad de decisión sobre su propia vida, aceptación de su diversidad, acceso a formación, a un empleo digno y a una vivienda, tratamientos integrales no coactivos que no alejen a la persona de su familia y entorno social, disfrute de la autonomía personal y una vida independiente, participación en el diseño y evaluación de políticas que les afectan, comprensión y apoyo, desarrollo de habilidades y técnicas para hacer frente al trastorno y a la situación familiar que de él se deriva.

2. La Ley General de Sanidad supuso la equiparación del trastorno mental al resto de problemas de salud, el fin de las instituciones psiquiátricas y el paso a la atención a los problemas de salud mental desde el ámbito comunitario, estipulando también que se pondrían en marcha "los servicios de reha-

bilitación y reinserción social necesarios para una adecuada atención integral (...) buscando la necesaria coordinación con los servicios sociales".
En definitiva, se han de procurar medidas que favorezcan la rehabilitación, recuperación e incorporación social y laboral, siendo un primer paso erradicar el estigma social que tanto afecta al colectivo y que influye negativamente en su recuperación.

\section{Medidas terapéuticas básicas y urgentes de atención para las personas con trastorno mental grave}

La atención al trastorno mental debe partir de que se asuma que los problemas de salud mental, entendidos como barreras al bienestar global al que cualquiera aspiramos, no están determinados solo por cuestiones biológicas e individuales. También juegan un papel fundamental los factores sociales, económicos, culturales, políticos y ambientales que obligan indudablemente a diseñar y ejecutar estrategias integrales y coordinadas para la promoción de la salud mental, prevención, tratamiento y recuperación en las que se impliquen múltiples perspectivas, agentes y administraciones.

En ocasiones, el trastorno mental puede ser pasajero y de mayor o menor complejidad. Sin embargo, cuando las personas presentan un trastorno mental grave y prolongado en el tiempo, escasa adherencia y/o acceso a los servicios de base comunitaria, hay una ausencia de recursos económicos, precariedad de alojamiento, sobrecarga familiar, elevado riesgo de recaídas y/o dificultades de adaptación al medio, se producen dificultades en su proceso de recuperación e inclusión social y laboral.

Esto lleva a que se tengan que aplicar mayores medidas de apoyo siempre respetuosas con los derechos reconocidos por la CIDPD (derecho a la vida, la libertad y seguridad, la dignidad, la salud, a vivir en comunidad, la educación, el acceso al empleo, etc.) y el resto de normativa vigente en materia de derechos y libertades. En el marco del XVII Congreso FEAFES "El reto de la coordinación sociosanitaria" (VV.AA., 20II), el Comité de Personas con Enfermedad 
Mental de FEAFES planteó una serie de medidas con las que atender los trastornos mentales adecuadamente y a la luz de la ESM aprobada por consenso de todas las comunidades autónomas en 2006 y revisada en 2009:

- Mantener vinculada a la persona con trastorno mental de su entorno social y familiar.

- Tratamientos en el entorno domiciliario (por ejemplo, asertivo comunitario y atención domiciliaria) y acompañamiento integral.

- Acciones sociocomunitarias integrales y coordinadas basadas en el modelo psicosocial para reducir las actuaciones coercitivas involuntarias.

- Inversión en psicoeducación.

Por otra parte, en febrero de 2013 un grupo de expertos mantuvo un encuentro de trabajo con representantes de FEAFES (personas con enfermedad mental, familiares y técnicos) en el que se concluyó que los recortes en las prestaciones sociales y el acceso a los servicios sanitarios ponían en riesgo la atención a las personas con enfermedad mental y sus familias. De aquella cita surgió un compendio de medidas terapéuticas básicas y cuya puesta marcha debía tener un carácter urgente.

Dichas medidas están en la línea de los planteamientos del Comité de Personas con Enfermedad Mental mencionados anteriormente y dan cumplimiento a la línea estratégica 2 de la ESM sobre "Atención a los trastornos mentales". Se trata de propuestas contempladas como recomendaciones y/o buenas prácticas en dicha Estrategia. Y su puesta en marcha podría garantizar, sin necesidad de grandes inversiones económicas por parte de la administración pública, un abordaje adecuado, integral, respetuoso con los derechos humanos, económicos, sociales y culturales, y enfocado a la recuperación y el bienestar global de la persona afectada. La implantación en todo el Estado supondría una reducción del gasto público en urgencias, en tratamiento farmacológico y en ingresos hospitalarios, garantizando esa sostenibilidad del Sistema
Nacional de Salud que todos deseamos y mejorando la calidad y seguridad de sus prestaciones.

- Equipo multidisciplinar: Constituyen la infraestructura básica del modelo comunitario de atención a los trastornos mentales y su función es esencial para ofrecer una atención integral, teniendo en cuenta la naturaleza bio-psicosocial de la salud y la diversidad de las personas. Su acción debe fundamentarse en los criterios de utilización de los tratamientos menos restrictivos posibles y de implicación de la persona con trastorno mental en las decisiones.

Este equipo, en el que la perfecta interacción entre sus miembros es esencial, para una población adulta de 30.000 habitantes debe estar formado por, al menos, I 4 profesionales: un psiquiatra, dos psicólogos/as (preferiblemente clínicos), cuatro enfermeros/as, un trabajador/a social, cuatro auxiliares de clínica, un terapeuta ocupacional, técnico de integración social, o similar, y un auxiliar administrativo.

- Plan Individualizado de Atención: Fundamentado en la necesidad de articular desde una primera intervención con la persona afectada un plan de atención individualizado reflejado por escrito en su historial clínico informatizado, que garantice un seguimiento y evaluación continuos. Este Plan se crea a partir de una reunión del equipo o del profesional con la persona con trastorno mental y su familia para establecer conjuntamente el seguimiento a realizar y nombrar a la persona que tutorizará el plan, que debe recoger cuatro aspectos:

- La evaluación de las necesidades de tratamiento, rehabilitación, cuidados y apoyos, y previsión de posibles situaciones de riesgo.

- La programación de todas las actuaciones necesarias para dar respuesta a estas necesidades y los 
criterios y plazos para la evaluación de su efecto.

- El acuerdo sobre esta programación entre el equipo de salud mental, los equipos de otros servicios que podrían estar involucrados en el caso, la persona con trastorno mental y/o su cuidador/a responsable.

- El nombre del miembro del equipo que será responsable de su ejecución y de las personas de referencia en cada uno de los dispositivos que la persona con trastorno mental grave pueda utilizar.

Esta medida terapéutica no requiere de una inversión económica específica y quedaría integrada en la metodología de trabajo a seguir por el equipo de profesionales encargado de apoyar a la persona con trastorno mental grave.

- Apoyo domiciliario: Podría prestarse a través de un programa de acompañamiento integral, de carácter terapéutico, individualizado e integral con el que favorecer la continuidad de la atención a las personas con trastorno mental y el desarrollo de su autonomía personal.

Este acompañamiento integral es preciso únicamente para personas con trastorno mental grave que presenten de forma consistente, o que estén en grave riesgo de tenerlas a corto o medio plazo, dificultades en aspectos básicos de la vida diaria, en el seguimiento de los tratamientos, en la continuidad de la atención sociosanitaria, en el acceso a los dispositivos y recursos para la integración social, en la participación de actividades en la comunidad.

Su meta es conseguir la recuperación, la integración y el acceso de las personas con trastorno mental grave a los recursos socio-comunitarios a partir de los apoyos necesarios. Promueve su autonomía personal y facilita el mantenimiento de una calidad de vida digna y el acceso a tratamientos integrales.

El programa de acompañamiento integral, fundamentado en el apoyo domiciliario, persigue específicamente:
- Favorecer la implicación de la persona con trastorno mental en su propio proceso de recuperación.

- Fomentar la participación de la familia en el acompañamiento del proceso de recuperación de su familiar.

- Potenciar el uso por parte de la persona con trastorno mental grave de los recursos sociales y sanitarios en la comunidad.

Para su implantación es preciso establecer cauces de colaboración entre los diferentes ámbitos implicados en su desarrollo y un equipo ejecutor formado por dos personas por cada Unidad de Salud Mental (un psicólogo clínico o trabajador social y un auxiliar de ayuda a domicilio).

- Equipo de Intervención Familiar: Todas las familias deben tener la oportunidad de acceder a una Intervención Familiar Psicoeducativa que incluya psicoeducación y entrenamiento en técnicas de comunicación interpersonal, de resolución de problemas y de prevención e intervención en crisis.

En los últimos veinte años se han realizado estudios con al menos 30 ensayos clínicos que demuestran que determinados métodos psicoeducativos llevados a cabo en el medio familiar mejoran significativamente el curso de la enfermedad, reduciendo notablemente el riesgo de recaídas al tiempo que se reduce la sobrecarga familiar, aspectos ambos de enorme trascendencia e impacto tanto entre las personas con trastorno mental grave como entre sus familias.

Como establecen las diferentes "Guías de Práctica Clínica” publicadas recientemente, intervenciones como la familiar psicoeducativa en los núcleos familiares donde hay una persona con trastorno mental grave, junto con otras intervenciones terapéuticas y tratamientos no basados únicamente en medicación, como son los entrenamientos en habilidades instrumentales y el tratamiento asertivo comunitario (modelo Gijón, modelo Avilés, etc.), contribuyen a mejorar el curso de la enfermedad y a prevenir las recaídas. 
Para el desarrollo de esta medida en una población de 250.000 habitantes se precisan únicamente dos profesionales de psicología clínica, trabajo social y DUE o auxiliar de enfermería, que hayan sido previamente formados específicamente en intervención familiar psicoeducativa por parte de la administración pública. Este equipo tendría que dedicar unas 40-60 horas para cada una de las familias de las 20-30 personas que, si la tendencia actual se mantiene, desarrollarán un trastorno mental grave.

\section{Conclusión. No hay excusas}

La Confederación FEAFES, como organización estatal representativa de las personas con enfermedad mental y sus familias y con la legitimidad que le confieren sus más de 30 años de experiencia defendiendo los derechos de este colectivo, prestando apoyo social e impulsando la elaboración de políticas públicas enfocadas a la plena recuperación de las personas con trastorno mental, recordando que no hay salud sin salud mental, mantiene su reivindicación sobre situar a las personas en el centro de cualquier tipo de atención. FEAFES apuesta de manera incondicional por el modelo comunitario en salud mental y alerta de las nefastas consecuencias que podría tener un hipotético distanciamiento de este modelo ${ }^{3}$.

FEAFES propone que las medidas terapéuticas básicas para atender a las personas con trastorno mental grave se incluyan en la Cartera Básica de Servicios del Sistema Nacional de Salud; se siga trabajando en el desarrollo e implantación de las recomendaciones establecidas en la ESM; se facilite la autonomía personal e inclusión social y laboral real; y se realice una adaptación normativa a las disposiciones de la CIDPD.

En definitiva, como sociedad no deberíamos permitir que la situación económica se convierta en una amenaza para la salud mental y el bienestar global de las personas. Sino que, de manera coordinada y transversal, deberían doblarse los esfuerzos por invertir de nuevo en sanidad pública, universal y gratuita, en fomento del empleo digno, en protección y apoyo social y en medidas especialmente dirigidas a los grupos más vulnerables, que detengan la cada vez mayor desigualdad y exclusión social.

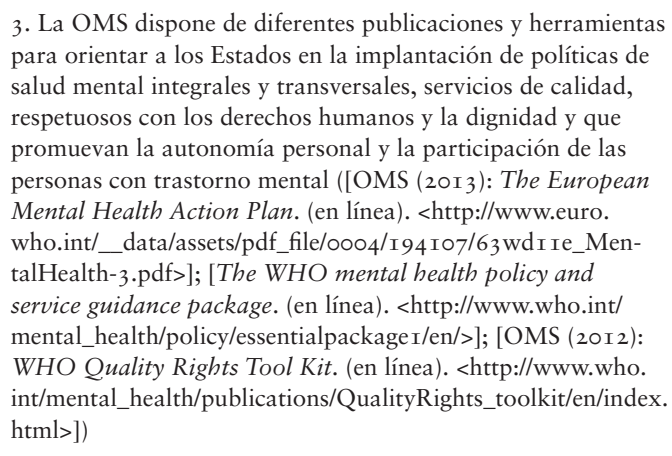




\section{Referencias bibliográficas}

España. Real Decreto Ley 20/20I2, de I3 de julio, de medidas para garantizar la estabilidad presupuestaria y de fomento de la competitividad, Boletín Oficial del Estado, núm. I 68 de I4 de julio de 2012 , p. 50462-50466.

Gómez Beneyto, M. (coord.) (20 I I): Estrategia en Salud Mental del Sistema Nacional de Salud 2009-2013, Madrid: Ministerio de Sanidad, Política Social e Igualdad.

Instituto Nacional de Estadística (20I4):

"Defunciones según la causa de la muerte. Año 20I 2". Nota de prensa (en línea). <http://www. ine.es/prensa/np830.pdf >, acceso 3 I de enero de 2014 .
Muižnieks, N (2013): Informe del Comisario para los derechos humanos del Consejo de Europa tras su visita a España del 3 al 7 de junio de 2013, Estrasburgo: Consejo de Europa.

Organización de Naciones Unidas (2006): Convención sobre los Derechos de las Personas con Discapacidad y Protocolo Facultativo, Nueva York: ONU.

Organización Mundial de la Salud (2013): Plan de acción sobre salud mental 20I3-2020, Ginebra: OMS.

VV.AA. (20I I): XVII Congreso FEAFES, El reto de la coordinación sociosanitaria (en línea). $<$ http://www.webcastlive.es/feafes20I I/\#>, acceso 5,6 y 7 de mayo de 20 I I. 